\title{
AVALIAÇÃO DE BLOCOS DE CONCRETO COM ADIÇÃO DE RESÍDUO DE VIDRO APLICADOS EM PAVIMENTAÇÃO*
}

\author{
Victor Barbosa de Souza ${ }^{1}$ \\ Niander Aguiar Cerqueira ${ }^{1}$ \\ André Luiz J. Alves ${ }^{1}$ \\ Aluísio P. Campbell ${ }^{1}$ \\ Anderson Flores Polonine ${ }^{1}$ \\ Amanda Camerini ${ }^{1}$ \\ Glênio Fernando Daniel ${ }^{1}$ \\ Fabio Da Costa Garcia Filho ${ }^{2}$ \\ Sérgio Neves Monteiro ${ }^{2}$
}

Resumo

Todos os anos toneladas de vidro são produzidos, sendo que restos de vidro são facilmente encontrados em resíduos domésticos e comerciais. $O$ resíduo de vidro ainda apresenta uma baixa taxa de reciclagem embora apresente um tempo estimado de decomposição de aproximadamente quatro mil anos. Este trabalho busca propor uma alternativa para resolver o problema da acumulação desses resíduos, através do uso desse material na construção de blocos de concreto, o que se apresenta como uma solução sustentável para redução do volume de vidro descartado. Neste trabalho, foi utilizado o resíduo de vidro moído, como agregado fino na mistura para a produção de pavimentos de cimento interligados, em substituição à areia em uma porcentagem de $25 \%$. A resistência à compressão e a absorção de água foram analisadas. Comparando os resultados dos blocos produzidos com o uso de vidro ao padrão (com a quantidade de areia normal), verificou-se que este novo produto apresenta alto potencial para contribuir para a reutilização desses resíduos nocivos para o meio ambiente, melhorando 0 desempenho dos blocos, reduzindo o custo e o consumo de matérias-primas.

Palavras-chave: Concreto; Sustentabilidade; Resíduo de Vidro; Blocos de Pavimentação.

\section{EVALUATION OF CONCRETE BLOCKS WITH ADDITION OF GLASS RESIDUE APPLIED IN PAVING}

\section{Abstract}

Tons of glass are produced annually, with scraps of glass from domestic and commercial waste being easily found. Glass waste still presents a low recycling rate, but an estimated time of decomposition of four thousand years, This work aims to present an alternative to solve the problem of accumulation of these residues, due the use of this material in the fabrication of concrete blocks, which would be a sustainable alternative to reduce the volume of discarded glass. In this work glass residue was used in substitution of sand, as a fine aggregate in the production of interlocked cement pavements, in a $25 \%$ ratio. The compressive strength and the water absorption were analyzed. Comparing the results of the blocks with the use of glass with standard $(100 \%$ sand), it was verified that this new product presents high potential to contribute to the reuse of these waste, thus can improve the performance of the blocks and reduce the cost and consumption of raw materials.

Keywords: Concrete; Sustentability; Glass waste; Paviment blocks. 


\section{INTRODUÇÃO}

Ao longo dos últimos 30 anos, aprendemos mais sobre o vidro e o seu processamento do que durante toda a história da tecnologia. Vidros são usados em quase todos os aspectos das atividades humanas, em casa, na ciência, na indústria e até na arte, uma vez que podem ser adaptados aos seus propósitos. A tecnologia desenvolvida e aplicada ao vidro permitiu adquirir novas vantagens em relação a outros materiais. Seu peso foi substancialmente reduzido, enquanto se tornou mais resistente, o vidro que invariavelmente era considerado de baixa resistência mecânica agora pode ser usado em novas aplicações, o que era inimaginável há poucas décadas atrás, e hoje graças às técnicas desenvolvidas, tratamentos térmicos e químicos é possível alcançar alta resistência, além de outras propriedades importantes para a fabricação de vários artefatos do vidro como parabrisas de carros, janelas à prova de balas, lentes de vidro, janelas e portas, recipientes para bebidas e entre outros [1-4].

O resíduo de vidro é um material com alto peso específico, que conta com superfícies de corte e poeira que são prejudiciais à saúde, tais fatores fazem com que seja associado um alto custo de transporte para uma fonte de reciclagem, além de exigirem um manuseio laborioso. Por esses motivos, muitas vezes o descarte do resíduo de vidro acaba ocorrendo de forma inadequada em lotes vazios, lixões, aterros sanitários, áreas rurais, entre outros lugares. A eliminação de resíduos incorretamente é um problema global, os aterros já são reduzidos e, com o passar dos anos, tendem a se sobrecarregar cada vez mais. Levando em consideração todos os materiais depositados na natureza, o grande vilão entre eles é o vidro, que leva cerca de 4000 anos a se decompor [5].

O vidro é um material $100 \%$ reciclável e amplamente utilizado em todo o mundo, as propriedades mecânicas incluem altos valores de resistência à compressão, resistência à tração, resistência à flexão e desgaste, possuindo alta resistência química e mecânica. Além disso, mais de $70 \%$ do material é constituído por sílica $\left(\mathrm{SiO}_{2}\right)$, que pode acarretar em boas propriedades pozolanas, o que o qualificaria como material constituinte potencial para a base de cimento. Considerando que o material de base cimentícia mais utilizado em obras para construção civil é o concreto, que é composto por uma mistura de agregados grosseiros e finos, aglutinante e água, podendo, inclusive, conter outros aditivos quando há necessidade de obter propriedades e características específicas. Tais características fazem com que esse material apareça como alternativa potencial para aplicação de resíduos de vidro, que permitiria a fabricação em grandes escala e com ampla aplicação em nosso país. Atuando como uma alternativa promissora para a fabricação de componentes e elementos de construção, o uso do resíduo de vidro como agregado pode atender a questões técnicas, econômicas, sociais e ambientais. E desta forma, ajudar a reduzir e a resolver o impacto ambiental dos resíduos de vidro, além de proporcionar um consumo reduzido de recursos naturais, reduzir a poluição e reduzir ainda mais o custo de fabricação de produtos compostos de concreto [6-8].

Podem-se imaginar várias formas possíveis de uso específico para a incorporação de resíduos de vidro, entre eles, destaca-se o pavimento interligado, que são peças pré-moldadas de concreto que têm como objetivo a utilização em pavimentação, sendo aplicadas em calçadas e ruas. Como essas peças não são usadas em obras estruturais, apenas para fins de pavimentação e superfícies de pisos, é seguro o uso dessas estruturas compostas. 


\section{MATERIAIS E MÉTODOS}

Para o procedimento experimental foram utilizados os cimentos CP V - ARI, areia natural, cascalho de basalto (esmagado $\mathrm{n}^{\circ} \# 0$ ) e resíduos de vidro. A partir destes foram produzidas duas misturas de concreto diferentes, o concreto normal usada na indústria local e outra com o uso do resíduo de vidro coletado. Os resíduos de vidro (Fig.1) utilizados no experimento foram coletados em lojas de vidro, serrarias e garrafas de vidro encontradas em toda a cidade, que haviam sido descartadas incorretamente na cidade de Itaperuna - RJ.

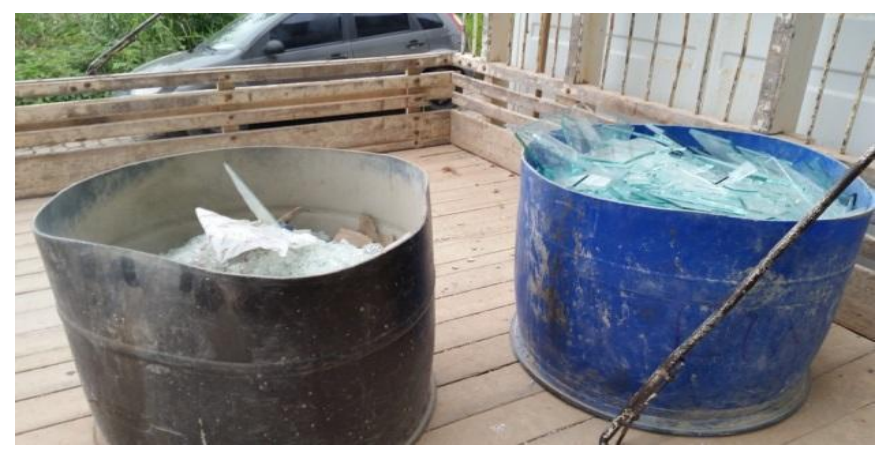

Figura 1. Resíduo de vidro coletado

Esses materiais foram escolhidos devido à sua abundância e não por não apresentarem um destino adequado na região. Após a coleta foi necessário reduzir as dimensões do resíduo, tornando-o mais próximo do tamanho de grãos de areia. A diminuição do tamanho do resíduo obtido inicialmente é feito através do esmagamento dos resíduos coletados, utilizando uma marreta, que resultou em diferentes tamanhos de grãos (Fig. 2 (a)).

Para obter um produto com dimensões consistentes com a areia utilizada, os fragmentos de vidro foram separados mecanicamente, usando uma peneira grosseira, o material passante obteve uma dimensão comparável à areia utilizada como agregado fino para o concreto como o uso do resíduo de vidro (Fig.2 (b)). Já para o concreto normal foi utilizado como agregado fino uma areia oriunda de Campos dos Goytacazes / RJ e, como agregado grosseiro, em ambas as formulações utilizou-se 0 cascalho $n \div 0$ de origem basáltica derivado da região de Muriaé / MG.

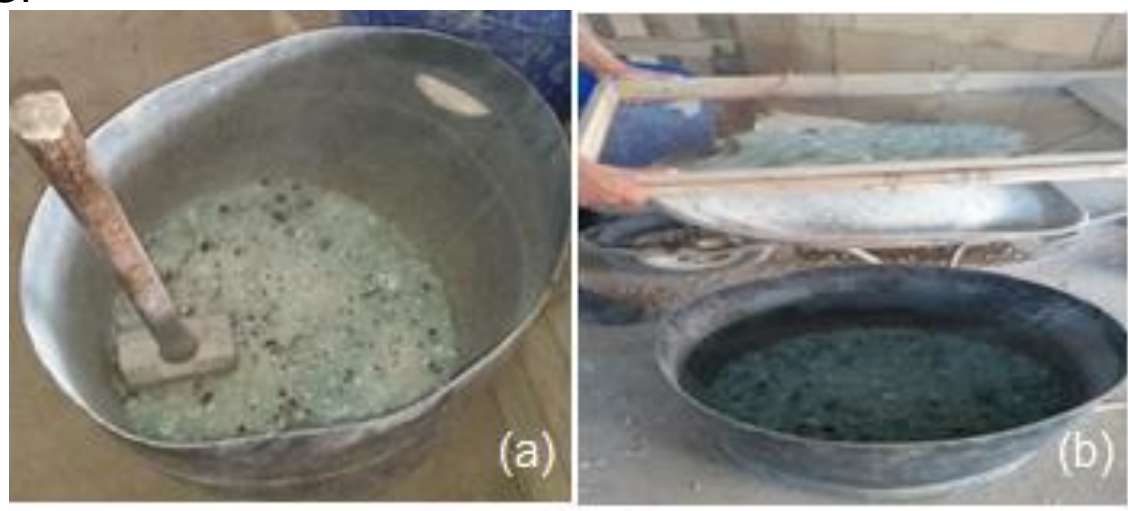

Figura 2. Esmagamento (a) e peneiração (b) do resíduo de vidro

Os blocos de concreto foram escolhidos de forma a apresentarem razão comprimento/largura de 2 e 16 faces, as dimensões dos blocos fabricados são 
apresentados na Tabela 1, tal configuração permite que a máquina de pavimentação, que usa esses blocos cuja a geometria é apresentada na Figura 3, seja montada em qualquer posição entrelaçando uns com os outros.

Tabela 1. Dimensionamento do bloco de concreto

\begin{tabular}{|c|c|c|c|}
\hline Largura $(\mathrm{mm})$ & Comprimento $(\mathrm{mm})$ & Profundidade $(\mathrm{mm})$ & Unidades / $\mathrm{m}^{\mathbf{2}}$ \\
\hline 110 & 220 & 80 & 39 \\
\hline
\end{tabular}

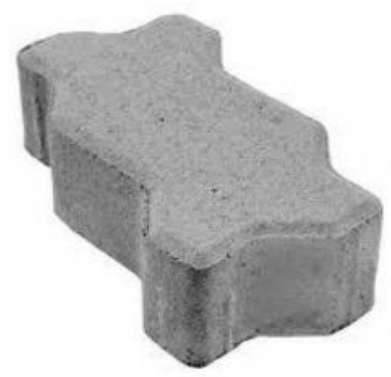

Figura 3. Geometria do bloco de concreto fabricado

A partir disso, foi estabelecida a porcentagem de mistura que seria utilizada para a produção do concreto. O procedimento para a produção dos blocos interligados seguiu a mesma metodologia utilizada para a linha de produção da fábrica, que além de nos permitir a utilização de suas instalações, nos forneceu sua força de trabalho para nos auxiliar no nosso trabalho. Essa escolha foi feita, com base no equipamento de moldagem que tem influencia direta nas características e qualidade dos blocos de pavimento.

Assim, foi preparada mistura de concreto com adição de resíduos de vidro e sem adição de resíduos - o padrão. A mistura para a produção de concreto com resíduos de vidro foi determinada a partir da mistura de concreto de referência, levando em consideração a proporção de $25 \%$ de substituição por volume de agregado fino (areia), pelo resíduo de vidro triturado e selecionado por peneiração. A fabricação de blocos de pavimentação realizada através em uma máquina hidráulica de vibração automática (Fig.4), adequada para produção em grande escala industrial que conta com uma capacidade de produção de cerca de $500 \mathrm{~m}^{2}$ / dia de concreto.

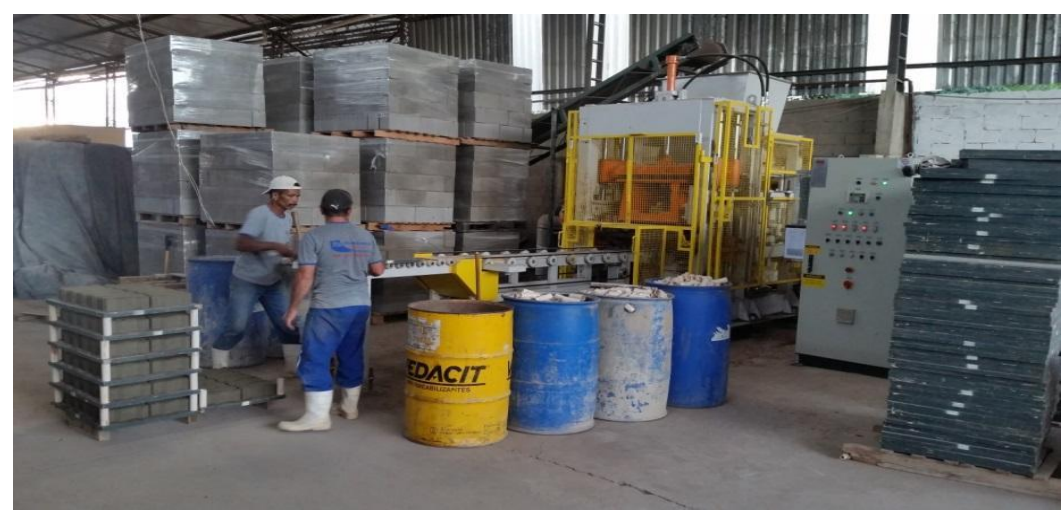

Figura 4. Máquina Hidráulica automática VibroPress

Foi realizada a cura úmida (Fig.5) por um período de sete dias. Posteriormente, foram selecionadas 12 amostras, sendo 6 referentes a mistura padrão e 6 para a mistura utilizando o resíduo de vidro, que foram separadas para realizar os ensaios de absorção e compressão dos blocos produzidos. 

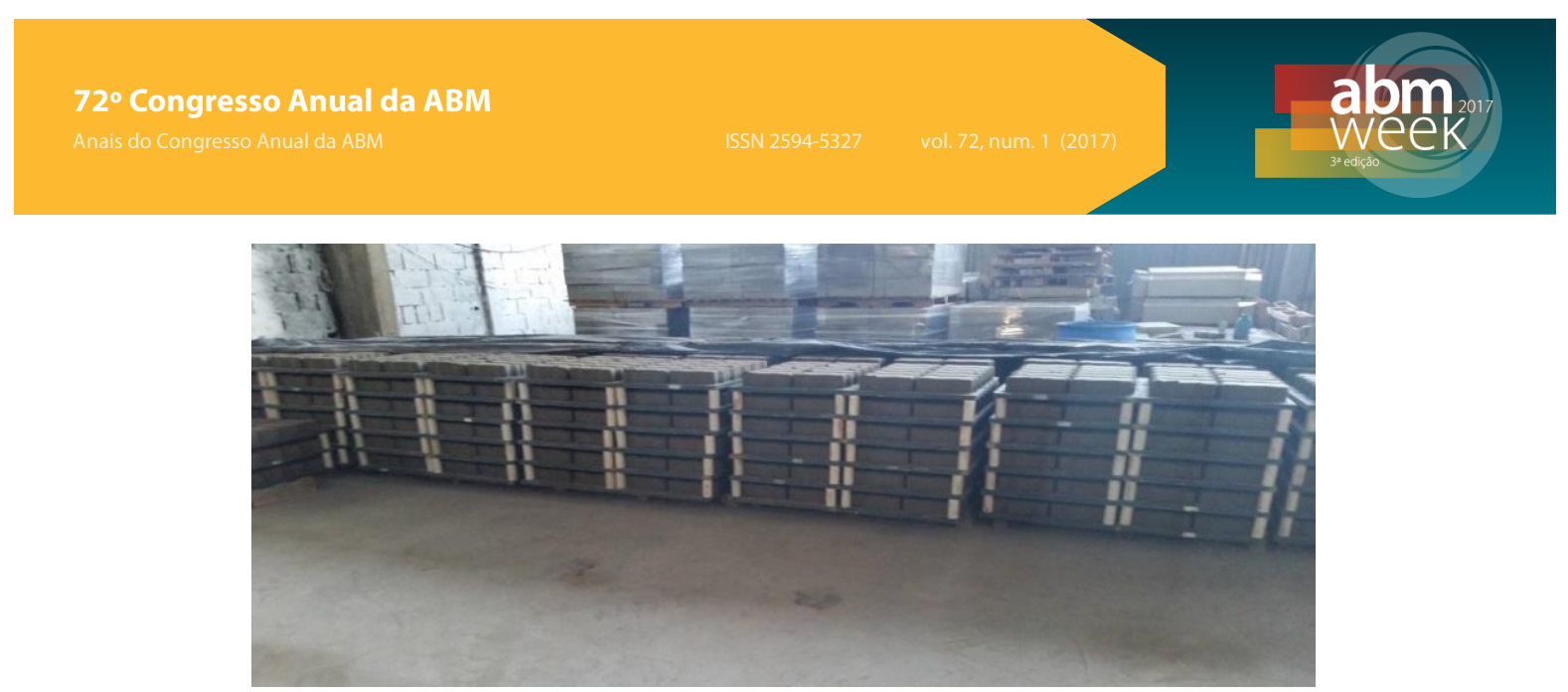

Figura 5. Blocos fabricados em processo de cura

O procedimento de determinação da resistência à compressão e absorção de água dos blocos de pavimentação foi baseado na norma ABNT NBR 9781: 2013 [9]. No teste de compressão foi utilizada uma prensa hidráulica da marca SOLOTEST, com capacidade para 100 toneladas de força, com eletrônica simples (indicador digital e bomba eletrohidráulica, que permite o controle manual da velocidade de avanço do pistão). $O$ teste indica a capacidade de carga suportada perpendicularmente pela peça, como mostrado na Figura 6, onde um bloco de pavimentação é comprimido uniaxialmente. Já o teste de absorção de água foi conduzido através da análise do incremento de massa de um corpo sólido poroso devido à entrada de água em seus poros permeáveis, em relação à sua massa seca, expressa em porcentagem. Todos os ensaios foram realizados no laboratório de Solo da UniRedentor em Itaperuna.

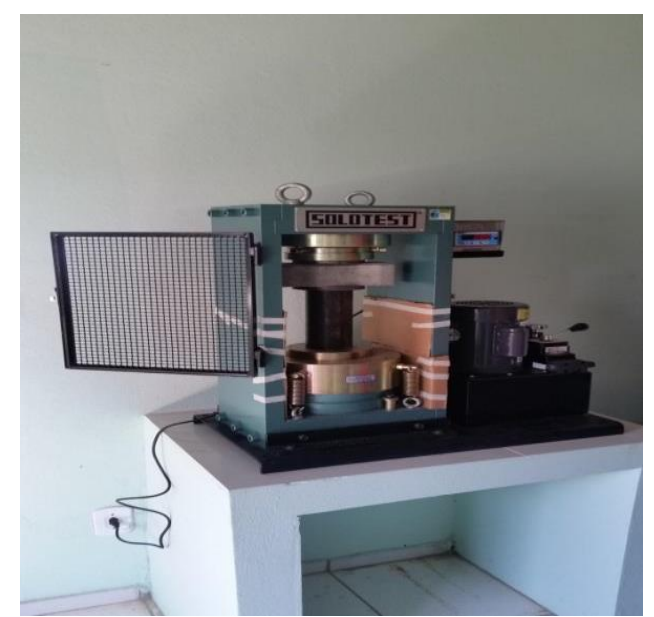

Figura 6. Prensa Hidráulica Uniaxial Solotest

\section{RESULTADOS E DISCUSSÃO}

Os testes de resistência à compressão individual dos blocos de pavimentação com uso de resíduos de vidro apresentaram valores maiores em comparação com os apresentados pela referência do pavimento interligado. Os pisos de composição padrão apresentaram o menor valor individual de 29,627 MPa e, por outro lado, os blocos de pavimentação com adição de resíduos de vidro apresentaram o maior com 41,27 MPa. Esses apresentaram uma média de resistência à compressão de $35,284 \mathrm{MPa}$, enquanto que para os blocos de composição padrão obtiveram média de 33,677 MPa. Em ambas as composições atingiram e até ultrapassam o mínimo de $28 \mathrm{MPa}$ para pisos com 7 dias de cura, e apresentaram $80 \%$ da resistência 
desejada de pelo menos $35 \mathrm{MPa}$ após 28 dias de cura, conforme prescrito na norma NBR 9781.

Quando estimamos a resistência à compressão dos lotes produzidos, temos que devido ao maior desvio padrão para os blocos fabricados com resíduos de vidro, a resistência estimada para o lote foi de $29,709 \mathrm{MPa}$, que quando comparado com os blocos de fabricação padrão (29,948 Mpa) representou um menor valor. Apesar disso, ambos os lotes apresentaram uma resistência estimada de pelo menos 1,709 MPa acima do mínimo especificado pela norma seguida.

Os resultados da análise da resistência mecânica à compressão são mostrados na Tabela 2 e no gráfico da Figura 7.

Tabela 2. Resistencia Mecânica à compressão dos blocos fabricados

\begin{tabular}{|c|c|c|c|c|c|}
\hline Amostras & Tons & $\begin{array}{c}\text { Compressão } \\
\text { máxima (Mpa) }\end{array}$ & $\begin{array}{l}\text { Média } \\
\text { (Mpa) }\end{array}$ & $\begin{array}{c}\text { Desvio } \\
\text { padrão (Mpa) }\end{array}$ & $\begin{array}{l}\text { Resistência a compressão } \\
\text { estimada do lote (MPa) }\end{array}$ \\
\hline \multirow{3}{*}{$\begin{array}{l}25 \% \\
\text { vidro }\end{array}$} & 30,45 & $\mathbf{3 1 , 4 3 3}$ & \multirow{3}{*}{35,284} & \multirow{3}{*}{5,25464} & \multirow{3}{*}{29,709} \\
\hline & 26,54 & 33,15 & & & \\
\hline & 39,98 & 41,27 & & & \\
\hline \multirow{3}{*}{$\begin{array}{l}100 \% \\
\text { areia }\end{array}$} & 23,72 & 29,627 & \multirow{3}{*}{33,677} & \multirow{3}{*}{3,51366} & \multirow{3}{*}{29,948} \\
\hline & $\mathbf{3 4 , 3 7}$ & 35,479 & & & \\
\hline & 34,8 & 35,923 & & & \\
\hline
\end{tabular}

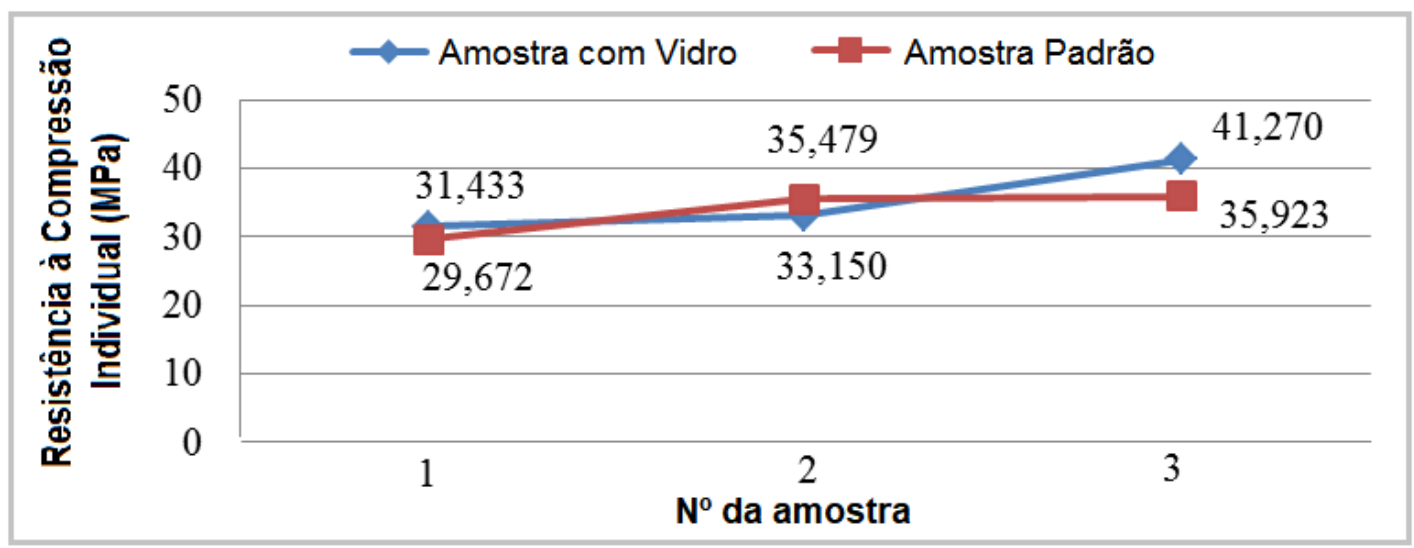

Figura 7. Resistência à compressão individual

Para o teste de absorção de água, foi possível observar uma maior absorção de água para blocos de pavimentação com resíduo de vidro na sua composição em relação ao produzido a partir da mistura padrão. Foi observado que o pavimento com vidro na sua composição tinha aproximadamente $5,114 \%$ de absorção média em relação à sua massa, enquanto que o de composição padrão apresentou uma absorção média de 3,504\% em relação à sua massa.

Para ambas as misturas os resultados obtidos foram satisfatórios, uma vez que ambos apresentaram a porcentagem de absorções médias e individuais abaixo do limite de 6\%, conforme determinado pela norma NBR 9781.

Os resultados da análise da porcentagem de absorção de água são apresentados na Tabela 3 e no gráfico da Figura 8. Os resultados obtidos a respeito da incorporação de resíduo de vidro nas propriedades de materiais de base cimentícia são coerentes com os encontrados na literatura. [10-11]

Tabela 3. Resultados de absorção de água dos blocos fabricados

\begin{tabular}{|c|c|c|c|c|}
\hline Amostra & Massa seca $(\mathrm{kg})$ & Massa úmida $(\mathrm{kg})$ & Absorção $(\%)$ & Média $(\%)$ \\
\hline V1 & $\mathbf{3 , 9 0 4}$ & $\mathbf{4 , 0 9 4}$ & $\mathbf{4 , 8 6 7}$ & \\
\hline
\end{tabular}




\begin{tabular}{|l|l|l|l|l|}
\hline V2 & $\mathbf{3 , 8 9 0}$ & $\mathbf{4 , 0 8 8}$ & $\mathbf{5 , 0 9 0}$ & \multirow{2}{*}{$\mathbf{5 , 1 1 4}$} \\
\cline { 1 - 3 } V3 & $\mathbf{3 , 7 8 8}$ & $\mathbf{3 , 9 9 2}$ & $\mathbf{5 , 3 8 5}$ & \\
\hline P1 & $\mathbf{4 , 0 0 4}$ & $\mathbf{4 , 1 2 8}$ & $\mathbf{3 , 0 9 7}$ & \multirow{2}{*}{$\mathbf{3 , 5 0 5}$} \\
\cline { 1 - 3 } P2 & $\mathbf{3 , 9 5 4}$ & $\mathbf{4 , 0 8 6}$ & $\mathbf{3 , 3 3 8}$ & \\
\hline
\end{tabular}

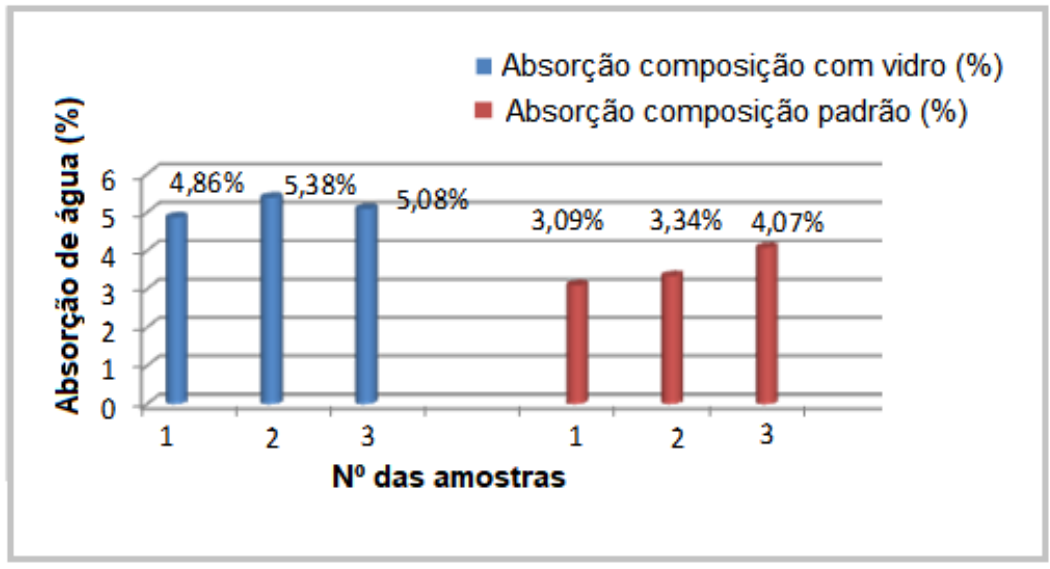

Figura 8. Análise da absorção de água dos blocos de concreto

\section{CONCLUSÃo}

- O bloco de pavimentação interligado de concreto com a incorporação de resíduos de vidro que substituem $25 \%$ da areia na composição padrão apresentou valores de resistência à compressão e valores de absorção de água dentro dos limites exigidos pelas normas brasileiras.

- O bloco de pavimentação com o vidro incorporado na composição apresentou o maior valor individual de resistência à compressão em comparação com os valores do bloco de pavimentação padrão.

- A incorporação de vidro no pavimento interligado da composição de concreto se apresenta como opção para o uso de resíduos de vidro na construção civil de maneira sustentável, para reutilizar o material de vidro que seria descartado de forma inadequada, reduzindo assim a disposição de vidro na natureza.

\section{Agradecimentos}

Os autores agradecem o apoio das agências brasileiras: CNPq, FAPERJ e CAPES para o desenvolvimento deste trabalho.

\section{REFERÊNCIAS}

1 ABIVIDRO. Disponível em: http://www.abividro.org.br/. Acesso em: 03/05/2017

2 Pinto TP. Metodologia para a gestão diferenciada de resíduos sólidos da construção urbana. 1999. Universidade de São Paulo. 
$3 \quad$ Vogel W. Chemistry of Glass. Ed. Springer-Verlag, Berlin. 1994.

4 Shao Y, Lefort T, Moraes S, Rodrigues D. Cement and Concrete Research. 30, 2000.

5 Fávero RB. Avaliação da utilização de sucata de vidro como adição mineral frente à sílica ativa e como agregado miúdo artificial na produção de materiais à base de cimento. 2009. Universidade Federal do Rio Grande do Sul.

6 Angulo SC, Ulsen C, Kahn H, John VM. Desenvolvimento de novos mercados para a reciclagem massiva de $\mathrm{RCD}$. V Seminário de Desenvolvimento sustentável e a reciclagem na construção civil. 2000; 293-308.

7 Shi C, Zheng K. A review on the use of waste glasses in the production of cement and concrete. Resource Conserv Recycl. 2007; 52; 234-247.

8 Tan K. Use of waste glass as sand in mortar: Part I - Fresh, mechanical and durability properties. Cement Concr Compos. 2011.

9 ABNT-ASSOCIAÇÃO BRASILEIRA DE NORMAS TÉCNINAS. NBR 9781: Peças de concreto para pavimentação- Especificações e métodos de ensaio. Rio de Janeiro, 2013.

10 Topçu IB, Canbaz M. Properties of concrete containing waste glass. Cement and Concrete Research. 2004; 34(1); 267-274.

11 López DAR, Azevedo CAP, Neto EB. Avaliação das propriedades físicas e mecânicas de concretos produzidos com vidro cominuído como agregado fino. Cerâmica. 2005; 51(320); 318-324. 